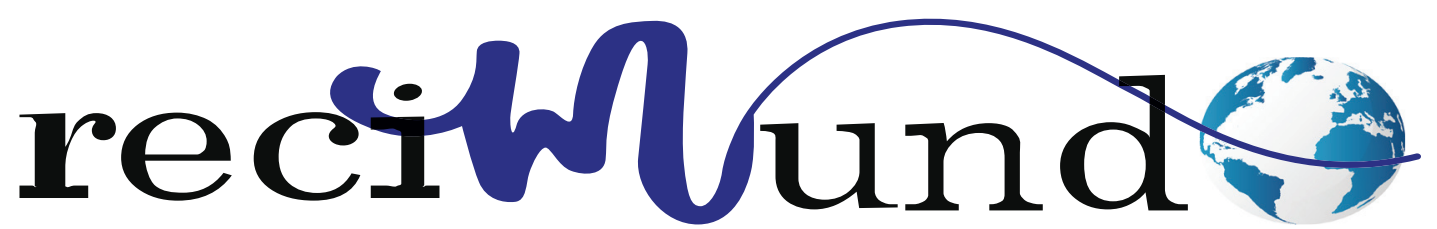

Revista Científica Mundo de la Investigación y el Conocimiento

DOI: 10.26820/recimundo/4.(1).esp.marzo.2020.200-209

URL: http://recimundo.com/index.php/es/article/view/799

EDITORIAL: Saberes del Conocimiento

REVISTA: RECIMUNDO

ISSN: 2588-073X

TIPO DE INVESTIGACióN: Artículo de Revisión

CÓDIGO UNESCO: 3205 Medicina Interna

PAGINAS: 200-209

\title{
Diagnóstico y tratamiento de la cetoacidosis diabética
}

\section{Diagnosis and treatment of diabetic ketoacidosis Diagnóstico e tratamento da cetoacidose diabética}

\section{Noemi Andrea Barrera Zambrano'; Andrea Isabel Ratti Torres; Denisse Lissette Flores Subia3; Karen Johanna Quiñonez García ${ }^{4}$}

\section{RECIBIDO: 20/11/2019 ACEPTADO: 29/01/2020 PUBLICADO: 05/03/2020}

1. Medico; Investigadora Independiente; Guayaquil, Ecuador; noemi_barrera15@hotmail.com; (D) https://orcid. org/0000-0002-7333-5149

2. Especialista en Medicina Interna; Medico; Investigadora Independiente; Guayaquil, Ecuador; andrearatti@hotmail.com; (D) https://orcid.org/0000-0002-9053-9303

3. Medico; Investigadora Independiente; Guayaquil, Ecuador; denisse64@hotmail.com; (D) https://orcid.org/00000001-7826-192X

4. Máster Universitario en Urgencias, Emergencias y Catástrofes; Médico; Investigadora Independiente; Guayaquil, Ecuador; nerak003@gmail.com; (D) https://orcid.org/0000-0002-9728-9642

CORRESPONDENCIA

Noemi Andrea Barrera Zambrano

noemi_barrera15@hotmail.com

Guayaquil, Ecuador 


\title{
RESUMEN
}

La cetoacidosis diabética abstracta (CAD), una complicación potencialmente mortal de la diabetes mellitus (DM), se produce más comúnmente en niños con diabetes tipo 1 de diabetes mellitus tipo 2. La hiperglucemia, acidosis, cetonemia, deshidratación y diversos electrolitos anomalías metabólicas como resultado de una deficiencia relativa o absoluta de insulina con o sin un exceso de hormonas contrarreguladoras. Gestión requiere una cuidadosa sustitución de déficit de líquidos y electrolitos, la administración intravenosa de insulina, y un estrecho seguimiento de parámetros clínicos y bioquímicos dirigidos a la detección oportuna de complicaciones, incluyendo hipopotasemia, la hipoglucemia y edema cerebral. El edema cerebral puede ser peligrosa para la vida y es administrado con la restricción de líquidos, la administración de manitol y soporte ventilatorio según se requiera. Factores que precipitan el episodio de cetoacidosis diabética deben ser identificados y rectificados. Después de la resolución de la cetoacidosis, la insulina intravenosa se la transición a vía subcutánea, titulando la dosis para lograr la normo glucemia.

Palabras clave: Edema Cerebral, Hiperglucemia, Terapia con Insulina, Cetosis Pediátrica.

\begin{abstract}
Abstract diabetic ketoacidosis (CAD), a life-threatening complication of diabetes mellitus (DM), most commonly occurs in children with type 1 diabetes mellitus type 2. Hyperglycemia, acidosis, ketonemia, dehydration and various electrolyte metabolic abnormalities such as result of a relative or absolute insulin deficiency with or without an excess of counter-regulatory hormones. Management requires careful replacement of fluid and electrolyte deficits, intravenous insulin administration, and close monitoring of clinical and biochemical parameters aimed at the timely detection of complications, including hypokalemia, hypoglycemia and cerebral edema. Cerebral edema can be life-threatening and is administered with fluid restriction, administration of mannitol and ventilatory support as required. Factors that precipitate the episode of diabetic ketoacidosis should be identified and rectified. After the resolution of ketoacidosis, intravenous insulin is transitioned into the subcutaneous route, titrating the dose to achieve normal blood glucose.
\end{abstract}

Keywords: Cerebral Edema, Hyperglycemia, Insulin Therapy, Pediatric Ketosis.

\section{RESUMO}

Resumo A cetoacidose diabética (DAC), uma complicação potencialmente fatal do diabetes mellitus (DM), ocorre mais comumente em crianças com diabetes mellitus tipo 1 tipo 2. Hiperglicemia, acidose, cetonemia, desidratação e várias anormalidades metabólicas eletrolíticas, como resultado de um parente ou deficiência absoluta de insulina com ou sem excesso de hormônios contra-reguladores. O manejo requer substituição cuidadosa dos déficits de líquidos e eletrólitos, administração intravenosa de insulina e monitoramento rigoroso dos parâmetros clínicos e bioquímicos, visando a detecção oportuna de complicações, incluindo hipocalemia, hipoglicemia e edema cerebral. O edema cerebral pode ser fatal e é administrado com restrição de fluidos, administração de manitol e suporte ventilatório, conforme necessário. Fatores que precipitam o episódio de cetoacidose diabética devem ser identificados e retificados. Após a resolução da cetoacidose, a insulina intravenosa é transferida para a via subcutânea, titulando a dose para atingir a glicemia normal.

Palavras-chave: Edema Cerebral, Hiperglicemia, Insulinoterapia, Cetose Pediátrica. 


\section{Introducción}

La cetoacidosis diabética (CAD) es una complicación importante de la diabetes mellitus, de ser la mayoría de las muertes relacionadas con la diabetes en los niños (Edge, Ford-Adams, \& Dunger, 2016). El diagnóstico es un reto, especialmente cuando los niños presentan con CAD en el inicio de la enfermedad. El monitoreo cuidadoso y la gestión adecuada son críticos con el fin de optimizar el resultado, prevenir complicaciones como edema cerebral y reducir la mortalidad. En este documento, se presenta un protocolo para la evaluación y gestión de la CAD en niños en base a las recomendaciones actuales.

La cetoacidosis diabética (CAD) en niños se define como la concentración de la hiperglucemia (glucosa sérica> 200-300 mg ( $\mathrm{dL}$ ) en presencia de acidosis metabólica ( $\mathrm{pH}$ arterial $<7.3$ con el nivel de bicarbonato sérico $<15 \mathrm{mEq} / \mathrm{L}$ ) y cetonemia (presencia de cetonas en la sangre) (Wolfsdorf, Glaser, \& Sperling, 2016). Como medición de cetonas en la sangre no está fácilmente disponible, cetonuria se utiliza como marcador de cetonemia. Cuando se mide, las cetonas de suero (de tasa hydroxybuty $B$ más acetoacetato) exceden $31 \mathrm{mg} / \mathrm{dl}$ con o sin cetonuria> 80 mg / dl (Sherry \& Levitsky, 2018). La cetoacidosis glicémica es observada en raras ocasiones, se producen en pacientes con vómitos prolongados, muy pobre ingesta oral, conocido diabetes mellitus tipo 1 (DM1) con insulina administrada antes de la visita de emergencia, y durante el embarazo. Con poca frecuencia, los adolescentes con diabetes mellitus tipo 2 (DM2) se pueden presentar con estado hiperosmolar hiperglucémico (HHS), definida por el azúcar en sangre $>600 \mathrm{mg} / \mathrm{dl}$ y el aumento de suero osmolalidad $>320 \mathrm{mOsm} / \mathrm{kg}$ en ausencia de acidosis significativa o cetonemia / cetonuria (Canarie \& Bogue, 2017).

La gravedad de la CAD se define por el grado de acidosis. CAD leve se define por el pH venoso entre 7,2 y 7,3 o bicarbonato de entre 10 y $15 \mathrm{mEq} / \mathrm{L}$; moderada por el $\mathrm{pH}$ entre 7,1 y 7,2 o bicarbonato de entre 5 y $10 \mathrm{mEq} / \mathrm{L}$; y grave por el $\mathrm{pH}$ venoso por debajo de 7,1 o bicarbonato por debajo de $5 \mathrm{mEq} / \mathrm{L}$ (Wolfsdorf, Glaser, \& Sperling, 2016).

\section{Metodología}

Para el desarrollo de este proceso investigativo, se plantea como metodología la encaminada hacia una orientación científica particular que se encuentra determinada por la necesidad de indagar en forma precisa y coherente una situación, en tal sentido (Davila, 2015) define la metodología "como aquellos pasos previos que son seleccionados por el investigador para lograr resultados favorables que le ayuden a plantear nuevas ideas".(p.66)

Lo citado por el autor, lleva a entender que el desarrollo de la acción investigativa busca simplemente coordinar acciones enmarcadas en una revisión bibliográfica con el fin de complementar ideas previas relacionadas al diagnóstico y tratamiento de la cetoacidosis diabética a través de una revisión de literatura, para así finalmente elaborar un cuerpo de consideraciones generales que ayuden a ampliar el interés propuesto.

\section{Tipo de Investigación}

Dentro de toda práctica investigativa, se precisan acciones de carácter metodológico mediante las cuales, se logra conocer y proyectar los eventos posibles que la determinan, así como las características que hacen del acto científico un proceso interactivo ajustado a una realidad posible de ser interpretada. En este sentido, se puede decir, que la presente investigación corresponde al tipo documental, definido por Castro (2016), "se ocupa del estudio de problemas planteados a nivel teórico, la información requerida para abordarlos se encuentra básicamente en materiales impresos, audiovi- 
suales y /o electrónicos". (p.41).

En consideración a esta definición, la orientación metodológica permitió la oportunidad de cumplir con una serie de actividades inherentes a la revisión y lectura de diversos documentos donde se encontraron ideas explicitas relacionadas con los tópicos encargados de identificar a cada característica insertada en el estudio. Por lo tanto, se realizaron continuas interpretaciones con el claro propósito de revisar aquellas apreciaciones o investigaciones propuestas por diferentes investigadores relacionadas con el tema de interés, para luego dar la respectiva argumentación a los planteamientos, en función a las necesidades encontradas en la indagación.

\section{Fuentes Documentales}

El análisis correspondiente a las características que predomina en el tema seleccionado, llevan a incluir diferentes fuentes documentales encargadas de darle el respectivo apoyo y en ese sentido cumplir con la valoración de los hechos a fin de generar nuevos criterios que sirven de referencia a otros procesos investigativos. Para (CASTRO, 2016) las fuentes documentales incorporadas en la investigación documental o bibliográfica, "representa la suma de materiales sistemáticos que son revisados en forma rigurosa y profunda para llegar a un análisis del fenómeno".(p.41). Por lo tanto, se procedió a cumplir con la realización de una lectura previa determinada para encontrar aquellos aspectos estrechamente vinculados con el tema, con el fin de explicar mediante un desarrollo las respectivas apreciaciones generales de importancia.

\section{Técnicas para la Recolección de la Infor- mación}

La conducción de la investigación para ser realizada en función a las particularidades que determinan a los estudios documentales, tiene como fin el desarrollo de un con- junto de acciones encargadas de llevar a la selección de técnicas estrechamente vinculadas con las características del estudio. En tal sentido, (Bolívar, 2015), refiere, que es "una técnica particular para aportar ayuda a los procedimientos de selección de las ideas primarias y secundarias". (p. 71).

Por ello, se procedió a la utilización del subrayado, resúmenes, fichaje, como parte básica para la revisión y selección de los documentos que presentan el contenido teórico. Es decir, que mediante la aplicación de estas técnicas se pudo llegar a recoger informaciones en cuanto a la revisión bibliográfica de los diversos elementos encargados de orientar el proceso de investigación. Tal como lo expresa, (Bolívar, 2015) "las técnicas documentales proporcionan las herramientas esenciales y determinantes para responder a los objetivos formulados y llegar a resultados efectivos" (p. 58). Es decir, para responder con eficiencia a las necesidades investigativas, se introdujeron como técnica de recolección el método inductivo, que hizo posible llevar a cabo una valoración de los hechos de forma particular para llegar a la explicación desde una visión general.

Asimismo, se emplearon las técnicas de análisis de información para la realización de la investigación que fue ejecutada bajo la dinámica de aplicar diversos elementos encargados de determinar el camino a recorrer por el estudio, según, (Bolívar, 2015) las técnicas de procesamiento de datos en los estudios documentales "son las encargadas de ofrecer al investigador la visión o pasos que debe cumplir durante su ejercicio, cada una de ellas debe estar en correspondencia con el nivel a emplear" (p. 123). Esto indica, que para llevar a cabo el procesamiento de los datos obtenidos una vez aplicado las técnicas seleccionadas, tales como: fichas de resumen, textual, registros descriptivos entre otros, los mismos se deben ajustar al nivel que ha sido seleccionado. 


\section{Resultados}

\section{Características clínicas}

Los niños con CAD clásicamente se presentan con poliuria, polidipsia, deshidratación, pérdida de peso, respiración de Kussmaul y aliento con olor a fruta. Sin embargo, la presentación puede ser neumonía mímica, asma, bronquiolitis aguda o abdomen. Se requiere un alto índice de sospecha para permitir el diagnóstico en niños sin antecedentes de diabetes. La inestabilidad hemodinámica o choque es raro como volumen intravascular se conserva a expensas de la deshidratación intracelular. Se debe estar alerta ante la aparición de edema cerebral, sobre todo si ya se ha iniciado la reposición de líquidos. La tabla 1 alista presentaciones comunes e indicadores importantes de la historia y el examen.

\section{Factores de Epidemiología y de riesgo}

El riesgo de presentación como la CAD en el inicio de la enfermedad varía inversamente con la incidencia de diabetes en la población, lo que refleja la dificultad en el reconocimiento de los síntomas de la diabetes en áreas con baja prevalencia de enfermedad. La CAD es la manifestación inicial de la diabetes en un 25\% (15-83\%) de los niños con DM1 y en 5-33\% de los niños con DM2 (Wolfsdorf, Glaser, \& Sperling, 2016). La CAD al momento del diagnóstico se encuentra más comúnmente en niños menores de 5 años de edad y los que pertenecen a las familias que no tienen acceso a la atención médica (Quinn, Fleischman, \& A., 2015).

El riesgo de la CAD en T1DM establecido es $1-10 \%$ por persona por año. La dosificación incorrecta o la omisión de la insulina es el factor precipitante la CAD en $75 \%$ de los casos; otros factores que predisponen a la CAD en niños con diabetes conocidos incluyen un mal control metabólico, episodios previos de la CAD, peri puberal y edad de los adolescentes con el sexo femenino, la presencia de trastornos de la alimentación, las circunstancias familiares difíciles o inestables, la falta de acceso a la atención médica y la falla de la bomba en niños tratados terapia con bomba de insulina (Hanas \& Lindgren, A 2-yr national population study of pediatric ketoacidosis in Sweden: predisposing conditions and insulin pump use., 2009). El riesgo también se incrementa durante tensiones tales como infecciones o cirugía. Anualmente, 15-20 niños presentan con CAD en el servicio de urgencias de nuestro centro; la mayoría de estos son los últimos niños diabéticos de comienzo.

\section{Fisiopatología de los trastornos de cier- tas bioquímicos CAD}

Un equilibrio entre la insulina y sus hormonas reguladoras de contador, incluyendo catecolaminas, glucagón, hormona del crecimiento y cortisol, mantiene suministro de combustible adecuado para el cerebro y otros tejidos durante los períodos de ayuno y estrés fisiológico. deficiencia relativa o absoluta de insulina (debido a la disminución de la producción de insulina o resistencia periférica a la acción de la insulina, en los tipos 1 y 2 diabetes, respectivamente), con o sin elevación de las hormonas contrarreguladoras en respuesta al estrés de una infección, trauma o cirugía, este equilibrio se inclina, produciendo un estado catabólico. El aumento de la producción de glucosa y la alteración de la utilización de glucosa periférica entonces conducen a hiperglucemia, hiperosmolaridad, glucosuria y diuresis osmótica. Incapacidad de los tejidos para utilizar la glucosa provoca la lipólisis con la producción de cuerpos cetónicos que son responsables por la acidosis metabólica, cetonemia, cetonuria y olor afrutado de acetona en el aliento. Durante la CAD, potasio intracelular se agota debido a los cambios causados por transcelular hipertonicidad y a cambio de protones que se han almacenado intracelularmente durante la acidosis metabólica. A su vez, este potasio se pierde debido a la hiperglucemia impul- 
sado diuresis osmótica, y con vómitos recurrentes. Hiperaldosteronismo secundaria a exacerba aún más las pérdidas de potasio agotamiento volumen. El fosfato es otro ion intracelular perdido del cuerpo debido a la diuresis osmótica.

\section{Gestión de la CAD}

La mayoría de los niños con CAD requieren ingreso. La reanimación inicial debe ir seguida de un seguimiento clínico y bioquímico frecuente. Los objetivos de la terapia en la CAD incluyen:

1. La corrección de la deshidratación

2. Corrección de la acidosis y la reversión de la cetosis

3. Restauración de la glucosa en sangre a cerca de lo normal

4. Evitar complicaciones de la terapia, edema cerebral particular

5. La identificación y el tratamiento del evento precipitante

6. La prevención de episodios recurrentes

La gestión de la CAD puede ser discutido en los siguientes apartados

\section{Evaluación inicial y Reanimación}

Un diagnóstico de la CAD se hace en presencia de la concentración de glucosa sérica> 200-300 mg / dl, pH de la sangre <7,3, el nivel de bicarbonato sérico $<15 \mathrm{mEq} / \mathrm{L}$ y cetonemia (Wolfsdorf, Glaser, \& Sperling, 2016). Como pruebas de diagnóstico rápido para la detección de cetonas en la sangre generalmente no están disponibles, la presencia de cetonas en la orina se busca.

El ingreso hospitalario está indicado en todos los casos, excepto aquellos con acidosis leve, sin deshidratación y la capacidad conservada tomar líquidos por vía oral. Las indicaciones para la admisión a la unidad de cuidados intensivos incluyen la presencia de graves factores de riesgo para la CAD o edema cerebral, tales como, la edad
$<5$ años y diabetes de nueva aparición. Cetoacidosis diabética severa es más común en niños con duración prolongada de los síntomas, la circulación comprometida y disminución del nivel de conciencia (Katz, 2003).

Un breve examen físico general basta para verificar el estado de las vías respiratorias, la respiración y la circulación y necesidad de reanimación (Tabla 1). Los pacientes con alteración de la conciencia requieren la vía aérea para ser asegurado; intubación y la colocación del tubo nasogástrico son esenciales si escala de coma de Glasgow cae por debajo

La oxigenación adecuada debe mantenerse el uso de oxígeno-flexible mental, según sea necesario mientras se monitoriza mediante pulsioximetría. Para los niños que presentan hipotensión, la reanimación con líquidos inmediata se realiza con solución salina normal al 0,9\% como 10-20 ml / kg en bolo dado rápidamente (Katz, 2003). Dos líneas de ánima intravenosas periféricas grandes están aseguradas, de los cuales una se mantendrá preferiblemente bloqueada para permitir el muestreo repetitivo de heparina sin dolor.

Si se conoce el peso de premórbida, peso actual ayuda a estimar el grado de deshidratación. Los signos en la exploración física son a menudo inexactos en la estimación de la gravedad de la deshidratación en estos niños. Debido a hiperosmolaridad, el volumen intravascular se conserva a expensas de la deshidratación intracelular. En presencia de recarga prolongada capilar, mucosa seca, ausencia de lágrimas, ojos hundidos, pulso débil y extremidades frías, o investigaciones que sugieren cetoacidosis moderada, se debe presumir moderado grado de deshidratación (pérdida de peso de 5-7\%). cetoacidosis grave puede suponer para indicar $7-10 \%$ de pérdida de peso, mientras que la hipotensión o volumen bajo / pulsos impalpables indican deshidratación grave

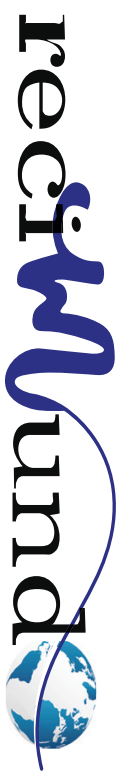


(pérdida de peso $\geq 10 \%$ ) (Hanas, Donaghue, $\&$ K., Clinical practice consensus guidelines 2006-2007, 2007).

\section{Evaluación bioquímica}

Si bien la obtención acceso intravenoso, los siguientes Investigación se envían para: glucosa sérica, electrolitos [de sodio $(\mathrm{Na}+)$, Potasio $(\mathrm{K}+)$, Calcio $(\mathrm{Ca}+)$, Magnesio (Mg2+), Fósforo (PO43-)], Gases en sangre venosa (pH, PCO2, HCO3-, Lactato, déficit de base), urea en sangre, creatinina, hematocrito y los recuentos totales y diferenciales de leucocitos. Si bien las cetonas en sangre no se miden de forma rutinaria, la orina se puede examinar fácilmente de cetonas de varilla. Si la medición de potasio en suero no está disponible o retardada, se obtiene un electrocardiograma (ECG) basal.

El grado de hiperglucemia puede predecir la gravedad de la deshidratación; glucosa en sangre> 500 mg / dl indica severidad.

La deshidratación por lo general acompañado por 30-40\% de reducción en la tasa de filtración glomerular (TFG), mientras que los niveles por encima de $800 \mathrm{mg} / \mathrm{dl}$ indican caída de la TFG en más del 50\%. A diferencia de sodio en el suero, elevaciones de nitrógeno ureico en sangre y el hematocrito se espera que se correlacionan con el grado de deshidratación. Aunque potasio corporal total es-de arrugado, los niveles séricos en la presentación pueden ser normal o alta debido a la redistribución en respuesta a la acidosis y la deficiencia de insulina.

La brecha en el nivel de sodio corregido, la osmolalidad calculada y el anión debe ser calculado como se sugiere en la Tabla 2, Ya que estos son útiles en la terapia de guía. Se espera que una amplia acidosis anión gap, típicamente entre 20 y 30 mmol / L; un anión gap > 35 sugiere acidosis láctica concomitante (Wolfsdorf, Glaser, \& Sperling, 2016). Como se ha detallado anteriormente, la hiponatremia observada es por lo general un artefacto. La osmolalidad efectiva de suero, que se calcula haciendo caso omiso de la contribución de urea libremente difusible a la osmolalidad total de, es a menudo entre 300 y 350 mOsm / L y se correlaciona bien con las anomalías en el estado mental (Fleckman, 2003).

Leucocitosis con desplazamiento hacia la izquierda es común en la CAD debido a la liberación de citoquinas y las catecolaminas, y no indica necesariamente la infección. Culturas (sangre y orina) y radiografía de tórax se obtienen si hay una sospecha de infección. La tomografía computarizada para evaluar el edema cerebral está prevista en caso de examen sugiere elevada tensión intracraneal.

\section{Reemplazo de agua y sal}

Cálculo del déficit de líquidos como se describió anteriormente, ya que las estimaciones clínicas de déficit de volumen son a menudo inexactas, hay que calcular los requerimientos de líquidos presumiendo un déficit de $30-50 \mathrm{ml} / \mathrm{kg}$ en leve a moderada CAD y 50-100 ml / kg en la CAD severa. Todos los fluidos intravenosos u orales dadas en otra ubicación deben tenerse en cuenta en los cálculos de fluidos.

Bolo de líquido si un paciente se presenta en estado de shock, $20 \mathrm{ml} / \mathrm{kg}$ de 0,9\% de solución salina (solución salina normal, NS) o Ringer's lactato se administra durante 15-30 minutos. Bolos de líquido adicional de 10-20 ml / kg NS y inotrópicos pueden administrarse si es necesario para restaurar el volumen circulatorio. Para los pacientes que se presentan con depleción de volumen grave sin choque, la reposición de volumen se inicia con NS a las 10-20 ml / kg / $\mathrm{h}$ durante 1-2 h. No se recomienda la administración de coloides.

Se recomienda que el 0,9\% de solución salina o de Ringer's acetato de ser utilizados 
en la inicial 4-6 h de la administración. Posteriormente, como la glucosa en la sangre se aproxima a $300 \mathrm{mg} / \mathrm{dl}$; o antes, si la caída de la glucosa en sangre es precipitada (Caída de> $90 \mathrm{mg} / \mathrm{dl} / \mathrm{h}$ ), el fluido que contiene $5 \%$ de dextrosa y con tonicidad entre $0.45 \%$ de solución salina a NS deben utilizarse. Sin embargo, si el suero de sodio es bajo (<132 mEq / L) y no se eleva con la caída en la glucosa en sangre, NS se puede utilizar para la reposición de líquidos posterior (Kitabchi \& Umpierrez, 2014).

Monitorización de la terapia de líquidos debe ser guiada por la evaluación clínica y cálculos en serie de la osmolalidad efectiva y sodio corregido. la reposición de líquidos exceso de celo, indicado por el cambio rápido en sodio corregido (> 1-2 mEq / L por hora) y la osmolalidad efectiva, se asocia con el desarrollo de edema cerebral (Wolfsdorf, Glaser, \& Sperling, 2016).

La velocidad de administración del fluido. El déficit de líquido total debe ser corregida de manera uniforme sobre 48 h a una velocidad de infusión que no exceda de 1,5-2 veces los requisitos de mantenimiento. Hay que tener en cuenta que restar cualquier líquido ya se administró en bolos (excepto para la reanimación) o justo antes de la visita de emergencia (Hillier, Abbott, \& RD., 2009).

\section{La terapia con insulina}

La terapia con insulina es esencial para revertir los daños metabólicas como la lipólisis y la cetogénesis, y para normalizar la glucosa en sangre. Terapia Timing con insulina se inicia después de la expansión de volumen inicial, es decir, 1-2 h después de comenzar la reposición de líquidos.

Bolo de administración de una dosis de bolo de insulina no se justifica. La disminución satisfactoria en la glucosa en suero se logra con la rehidratación sola, y el uso de bolos se asocia con la aparición de edema cerebral (Glaser \& Barnett, 2011).

Dosis bajas de la terapia de insulina intravenosa a 0.1 Unidad por kg por hora es el estándar de cuidado. Las dosis más altas se asocian con un mayor riesgo de hipopotasemia, hipoglicemia y una disminución demasiado rápida de la osmolalidad en suero, mientras que las tasas más bajas pueden ser inadecuados para reprimir la cetogénesis.

El cebado de la tubería de cebado de la tubería debe ser realizada por el lavado de solución de insulina a través de la tubería antes de la infusión en el paciente, porque se une de insulina para botellas de vidrio, bolsas IV de plástico, jeringas y tubos.

Duración de la terapia con insulina regular se administra a la misma tasa $(0,1 \mathrm{U} / \mathrm{kg} /$ h) hasta la resolución de la cetoacidosis, es decir, pH venoso> 7,3, HCO3- > 15 mmol / L y el cierre de la brecha de aniones).

Ajuste de la dosis después de inicio de la terapia con insulina, se espera que la glucosa en sangre a caer a una velocidad de 36-90 mg / dl / h (Kappy, Lightner, \& ES, 1999). Desde la normalización de glucosa en sangre se produce mucho antes resolución de la cetoacidosis, la concentración de dextrosa en el líquido de sustitución debe aumentarse según sea necesario para mantener la glucosa entre 150-200 mg / dl. Si la hipoglucemia ocurre a pesar de aumento de la fuerza de solución de dextrosa hasta $12,5 \%$ (a partir de 5\%), la dosis de insulina puede reducirse en decrementos de 0,02 unidades / kg / h hasta 0,05 unidades / kg / h, siempre que la acidosis metabólica continúa resolviendo. Los niños pequeños con la CAD, los niños mayores con diabetes establecidos y los que tienen estados hiperosmolares no cetóticos tienden a demostrar marcada sensibilidad a la insulina. Algunos sugieren el uso de sistema de infusión de dos bolsas, en el que las soluciones electrolíticas idénticas que difieren sólo en la con-

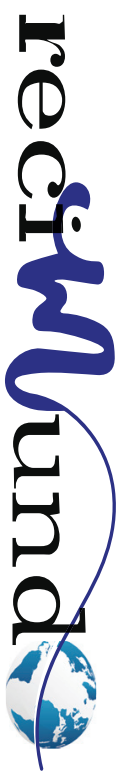


centración de dextrosa (uno en el 0\%, otro a 10\%) se ejecutan de forma simultánea a través de diferentes cánulas, con las tasas de administración tituladas como por resultados de sangre prueba de glucosa (Grimberg \& Cerri, 2009).

La resistencia a la insulina se sospecha si la tasa de caída de glucosa en sangre <70 mg / dl / h o acidosis no está mejorando a pesar de que la glucosa en sangre está cayendo. Velocidad de infusión se puede aumentar gradualmente a $0.3 \mathrm{U} / \mathrm{kg} / \mathrm{h}$ después de descartar errores en la prescripción de la insulina o de la preparación y la extravasación de la línea intravenosa.

\section{Reemplazo de potasio}

La adición de potasio a los fluidos intravenosos es esencial en la CAD debido a un déficit real en el potasio corporal total de alrededor de 3-6 mEq / kg, principalmente de la piscina intracelular. El potasio debería añadirse al fluido a una velocidad de 40 $\mathrm{mEq} / \mathrm{L}$ (idealmente 50\% como cloruro y $50 \%$ como fosfato) una vez que el niño ha anulado y un nivel sérico normal ( $<6 \mathrm{mEq} /$ L) se ha documentado, por lo general concurrente con iniciar la terapia con insulina (Fleckman, 2003).

Si el paciente es hipopotasémico, el reemplazo de potasio se inicia en el momento de la expansión de volumen inicial incluso antes de comenzar la terapia de insulina. Si el paciente es hipercalórico, la terapia de reemplazo de potasio se difiere hasta que se documenta la producción de orina.

La tasa máxima recomendada de reemplazo intravenoso de potasio es de 0,5 mEq / kg / h (o 80 mEq fluido intravenoso / L) (Glaser \& Barnett, 2011). Esto requiere un seguimiento cuidadoso. Si persiste hipocalemia pesar de una tasa máxima de reposición de potasio, entonces la tasa de infusión de insulina se puede reducir.

\section{Supervisión}

El manejo exitoso de la CAD requiere una documentación meticulosa de la respuesta clínica y bioquímica a la terapia, en un diagrama de flujo. La tabla 2 proporciona la frecuencia deseable de cada observación.

La evaluación continua de los signos vitales en pacientes críticamente enfermos es facilitada por el uso de un monitor cardíaco y la colocación de un catéter arterial para la monitorización de la presión arterial invasiva. Mientras taquicardia persistente e hipotensión indican continua hipovolemia, una caída en la frecuencia cardíaca con el nuevo desarrollo de la hipertensión sugiere aumento de la presión intracraneal, como se ve con edema cerebral. Los pacientes con depresión de la conciencia deben ser cateterizados para permitir la cartografía producción de orina por hora precisa. El personal de enfermería debe registrar cuidadosamente todos los fluidos recibida, incluyendo bolos de solución salina, el volumen administrado con medicamentos inyectables o como infusiones, y cualquier ingesta oral.

\section{Conclusiones}

La cetoacidosis diabética es una causa importante de morbilidad y mortalidad en los niños con diabetes mellitus. El diagnóstico oportuno, la gestión apropiada, monitoreo cuidadoso y complicaciones aprehender son fundamentales para garantizar un resultado favorable. Tratamiento del edema cerebral es un reto y el resultado sigue siendo insatisfactoria. Estrategias para una adecuada gestión de esta complicación requieren investigación adicional.

Infecciones raramente precipitan CAD. En los pacientes previamente diagnosticados con diabetes, la omisión de la insulina, ya sea deliberada o accidental, subyace a $75 \%$ casos de la CAD. Se debe identificar y tratar los factores subyacentes este fenómeno, in- 
cluyendo una mala situación socioeconómica, la falta de acceso a servicios de salud y las preocupaciones psicosociales, como la falta de supervisión de los padres, trastornos de la alimentación, problemas psiquiátricos y conceptos erróneos como la retención de la insulina durante el estrés, tales como el hambre, vómitos o infecciones.

Los pacientes recientemente diagnosticados y sus padres deben recibir instrucciones relativas a la administración de insulina, control de glucosa en casa y cuidado de día de enfermedad. Un proveedor de atención de salud debe ser de fácil acceso en caso de emergencia. Conciencia entre los médicos es esencial para permitir el reconocimiento y la gestión de la inminencia de la cetoacidosis diabética temprana, por lo que la admisión hospitalaria, complicaciones como edema cerebral, y la consiguiente morbilidad y la mortalidad se pueden evitar.

\section{Bibliografía}

Bolívar, J. (2015). Investigación Documental. México: Pax.

Canarie, M., \& Bogue, C. (2017). Decompensated hyperglycemic hyperosmolarity without significant ketoacidosis in the adolescent and young adult population. J Pediatr Endocrinol Metab, 20(10), 1115-24. Obtenido de https://www.ncbi.nlm.nih. gov/pubmed/18051930

Castro, J. (2016). Técnicas Documentales. México: Limusa.

Davila, A. (2015). Concepto de terminos cientificos. Caracas: Oasis.

Edge, J., Ford-Adams, M., \& Dunger, D. (2016). Causes of death in children with insulin-dependent diabetes 2010-16. Arch Dis Child, 318-23.
Fleckman, A. (2003). Diabetic ketoacidosis. Endocrinol Metab Clin North Am. , 181-207.

Glaser, N., \& Barnett, P. (2011). Risk factors for cerebral edema in children with diabetic ketoacidosis: the Pediatric Emergency Medicine Collaborative Research Committee of the American Academy of Pediatrics. N Engl J Med., 264-9.

Grimberg, A., \& Cerri, R. (2009). The "two bag system" for variable intravenous dextrose and fluid administration: benefits in diabetic ketoacidosis management. . J Pediatr., 376-8.

Hanas, R., \& Lindgren, F. (2009). A 2-yr national population study of pediatric ketoacidosis in Sweden: predisposing conditions and insulin pump use. Pediatr Diab., 33-7.

Hanas, R., Donaghue, \& K. (2007). Clinical practice consensus guidelines 2006-2007. Pediatr Diab, 341-2.

Hillier, T., Abbott, \& RD. (2009). Hyponatremia: evaluating the correction factor for hyperglycemia. Am J Med, 399-403.

Kappy, M., Lightner, \& ES. (1999). Low-dose intravenous insulin in the treatment of diabetic ketoacidosis. . Am J Dis Child. , 523-5.

Katz, M. (2003). Hyperglycemia-induced hyponatremia: Calculation of expected serum sodium depression. . N Engl J Med., 843-4.

Kitabchi, A., \& Umpierrez, G. (2014). Hyperglycemic Crises in Adult Patients With Diabetes. Diabetes Care, 32(7), 1335-1343. doi:10.2337/dc09-9032

Quinn, M., Fleischman, \& A. (2015). Characteristics at diagnosis of type 1 diabetes in children younger than 6 years. J Pediatr, 148(3), 366-71. Obtenido de https://www.ncbi.nlm.nih.gov/pubmed/16615969

Sherry, N., \& Levitsky, L. (2018). Management of diabetic ketoacidosis in children and adolescents. . Pediatr Drugs., 209-15.

Wolfsdorf, J., Glaser, N., \& Sperling, M. (2016). Diabetic ketoacidosis in infants, children, and adolescents. A consensus statement from the American Diabetes Association. Diab Care., 1150-9.

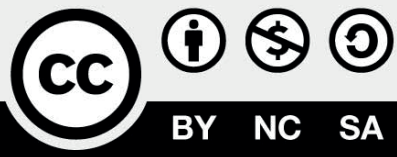

RECONOCIMIENTO-NOCOMERCIAL-COMPARTIRIGUAL CC BY-NC-SA

ESTA LICENCIA PERMITE A OTROS ENTREMEZCLAR, AJUSTARY CONSTRUIR A PARTIR DE SU OBRA CON FINES NO COMERCIALES, SIEMPRE Y CUANDO LE RECONOZCAN LA AUTORÍA Y SUS NUEVAS CREACIONES ESTÉN BAJO UNA LICENCIA CON LOS MISMOS TÉRMINOS.

\section{CITAR ESTE ARTICULO:}

Barrera Zambrano, N., Ratti Torres, A., Flores Subia, D., \& Quiñonez García, K. (2020). Diagnóstico y tratamiento de la cetoacidosis diabética. RECIMUNDO 4(1(Esp)), 200-209. doi:10.26820/recimundo/4.(1).esp.marzo.2020.200-209 